[0212-7199 (2007) 24: 7; pp 335-338] ANALES DE MEDICINA INTERN Copyright (C) 2007 ARAN EDICIONES, S.L.

AN. MED INTERnA (Madrid) Vol. 24, N. ${ }^{\circ}$, pp. $335-338,2007$

\section{Hiponatremia sintomática secundaria a tratamiento conjunto anticomicial y antidepresivo: ¿riesgo de muerte súbita en epilepsia?}

\author{
M. A. RUIZ GINÉS, S. GARCÍA GARCÍA', J. A. RUIZ GINÉS², E. TZE KIONG³ \\ E. FERNÁNDEZ RODRÍGUEZ
}

Servicios de Análisis Clínicos y Bioquímica y ${ }^{\prime}$ Urgencias. Hospital Virgen de la Salud. Toledo. ${ }^{2}$ Servicio de Neurología. Hospital Universitario de la Princesa. Madrid. ${ }^{3}$ Kaohsiumg Medical University Hospital. Taiwan
SYMPTOMATIC SECONDARY HYPONATRAEMIA DUE TO COMBINED TREATMENT ANTICONVULSANT AND ANTIDEPRESSANT: RISK OF SUDDEN DEATH IN EPILEPSY?

\section{RESUMEN}

La notable incidencia de epilepsia en nuestro medio, con la presencia, cada vez más frecuente, de refractariedad terapéutica, obliga a la politerapia anticomicial. Si partimos del hecho, de que muchas de las epilepsias tienen una base secundaria, con trastornos comportamentales asociados, podemos comprender la cada vez más habitual asociación entre fármacos anticomiciales y antidepresivos.

Presentamos el caso clínico de una mujer de 37 años, afecta de epilepsia farmacorresistente asociada a síndrome depresivo exógeno, en tratamiento conjunto con Carbamacepina, Lamotrigina y Venlafaxina que sufre cuadro sincopal secundario a hiponatremia sintomática, cuya patogénesis radica en un mecanismo de secreción inadecuada de hormona antidiurética (SIADH).

La relevancia de este caso radica en el hecho de que la hiponatremia generada por esta combinación farmacológica, hasta ahora, no ha sido descrita en la literatura. Esta anomalía electrolítica puede derivar en efectos secundarios neurológicos y cardiovasculares, en ocasiones tan relevantes, como para producir el fenómeno conocido como muerte súbita en el paciente epiléptico (SUDEP).

Recomendamos, por ello, un estricto control iónico en aquellos pacientes en tratamiento conjunto anticomicial y antidepresivo, a fin de evitar episodios paroxísticos vasculares y minimizar el riesgo de SUDEP.

PALABRAS CLAVE: Hiponatremia. Carbamacepina. Lamotrigina. Venlafaxina. Epilepsia refractaria. Síncope. Muerte súbita en epilepsia. Selenio. Neuroprotección.
ABSTRACT

The notable incidence of epilepsy in the general population, with the presence, more and more frequent of refractory therapeutic, it forces the anticonvulsant polytherapy. If we depart from the fact, that many of the epilepsies have a secondary cause, with affective disorders associate, we can understand the most habitual association of anticonvulsant and antidepressant.

We present the clinical case of a 37-year-old-woman with refractory therapeutic epilepsy associated to exogenous depressive syndrome, in combined treatment with Carbamazepine, Lamotrigine and Venlafaxine, that suffers a episode of syncope secondary to symptomatic hyponatraemia, generated by syndrome of inappropriate secretion of antidiuretic hormone (SIADH).

The relevance of this case resides in the fact that the hyponatraemia generated by this pharmacological combination, up to now, it has not been described in the literature.

This electrolytic anomaly can derive in secondary neurological and cardiovascular effects, in so outstanding occasions, as to produce the phenomenon denominated sudden death in the epileptic patient (SUDEP).

We recommended a strict ionic control in those patients that require anti-epileptic and anti-depressant treatment combined, in order to avoiding paroxistic vascular episodes and to minimize the risk of SUDEP.

KEY WORDS: Hyponatraemia. Carbamazepine. Lamotrigine. Venlafaxine. Epilepsy. Syncope. Sudden death. Selenium. Neuroprotections.

Ruiz Ginés MA, García García S, Ruiz Ginés JA, Fernández Rodríguez E. Hiponatremia sintomática secundaria a tratamiento conjunto anticomicial y antidepresivo: ¿riesgo de muerte súbita en epilepsia? An Med Interna (Madrid) 2007; 24: 335-338.

\section{INTRODUCCIÓN}

La muerte súbita en la epilepsia (sudden death in epilepsySUDEP) es una grave complicación que está alcanzando notable interés dada la prevalencia de dicha enfermedad en nuestro medio.

Ya en 1868, Bacon (1), consideró varias causas de muerte en pacientes epilépticos, a saber:

1. Dependientes de la evolución de la enfermedad, dado su carácter crónico.
2. Resultado de episodios comiciales sucesivos.

3. Muerte inesperada ("súbita") en el seno de una convulsión. 4. Secundaria a accidentes durante una crisis comicial.

Desde entonces, diferentes autores se han pronunciado al respecto, así Oppenheimer (1990) (2) la definió como aquella muerte repentina de un paciente epiléptico, por otra parte sano, coincidiendo con una crisis o sospecha de crisis y en ausencia de traumatismo, estado de mal epiléptico, aspiración u otra causa identificable. 
Nashef (1997) (1) define SUDEP como "muerte súbita e inesperada, presenciada o no por testigos, no traumática ni secundaria a inmersión, en pacientes epilépticos con o sin evidencias de haber sufrido crisis epiléptica al morir (excluyendo el estado de mal epiléptico documentado), con examen postmortem que no muestre factores tóxicos o anatómicos que expliquen la muerte".

A partir de las diferentes definiciones se derivan una serie de criterios necesarios para el diagnóstico de SUDEP, como son:

1. La víctima debe tener un diagnóstico previo de epilepsia.

2. Muerte inesperada de forma súbita.

3. Puede o no existir evidencia de una crisis epiléptica al morir, pero nunca presencia de status.

4. Debe descartarse cualquier causa de muerte accidental o natural.

La epilepsia "per se" conlleva mayor riesgo de mortalidad. Analizando la tasa de mortalidad de los pacientes epilépticos, se observa que es del orden de dos a tres veces superior a la de la población general (3). Se ha estimado que la incidencia de muerte súbita (SUDEP) se produce en 1:1.000 pacientes epilépticos (4), constituyendo del $8-17 \%$ de las muertes en pacientes epilépticos. Esta cifra se incrementa en las epilepsias refractarias al tratamiento.

Se consideran como factores de riesgo de SUDEP: sexo masculino, adultos jóvenes (30-40 años), episodios tónicoclónicos generalizados, déficit neurológico concomitante, alcoholismo, crisis durante el sueño, crisis en ausencia de testigos o refractarias al tratamiento farmacológico, epilepsias de comienzo precoz, más de 10 años de evolución, necesidad de politerapia, períodos de transición farmacológica, con bajos o altos niveles plasmáticos de anticonvulsivantes, mal control farmacológico, tratamiento concomitante con antidepresivos y antipsicóticos.

Fármacos antiepilépticos como la Carbamazepina o la Fenitoína han sido considerados como posible causa desencadenante de SUDEP (5), extendiéndose a otros antiepilépticos, como la Lamotrigina, todos ellos en relación con un alargamiento del intervalo QT (6-8).

El mecanismo fisiopatológico más relevante relacionado con SUDEP es la descarga $\alpha$-adrenérgica central que puede llegar a originar edema pulmonar, apnea central (hipoxemia y lactato elevado) o alteraciones del ritmo cardiaco causado por una disfunción autonómica (bradicardia, bloqueo sinusal y prolongación del intervalo QT, así como en pacientes con severa resistencia a tratamiento antiepiléptico pueden llegar a presentar episodios de cambios en el segmento ST).

En España, hasta la fecha, se han publicado sólo dos casos de hiponatremia sintomática por Carbamazepina, uno debido a la interacción entre Carbamazepina y Claritromicina y otro por Carbamazepina y Paroxetina $(9,10)$.

Presentamos el caso clínico de una paciente afecta de epilepsia farmacorresistente y depresión secundaria, en tratamiento conjunto con Carbamacepina, Lamotrigina y Venlafaxina, que desarrolla una hiponatremia sintomática. Su finalidad es dar a conocer la importancia de una adecuada valoración riesgo-beneficio a la hora de prescribir asociaciones farmacológicas potencialmente hiponatremiantes, así como la necesidad de establecer controles estrictos de iones y niveles de drogas en sangre. Todo ello, con el fin último de prevenir complicaciones mayores asociadas a dicha alteración hidroelectrolítica, entre la que cabe destacar, por su gravedad, el SUDEP.

\section{CASO APORTADO}

Mujer de 37 años, sin alergias medicamentosas conocidas. Factores de riesgo cardiovascular: Dislipemia en tratamiento con Pravastatina $10 \mathrm{mg}$. No refiere hábitos tóxicos. Encefalitis por varicela diagnosticada a los 27 años, con crisis comiciales parciales complejas secundariamente generalizadas sintomáticas desde entonces. Actualmente en tratamiento con Carbamacepina $400 \mathrm{mg}$, a dosis de $800 \mathrm{mg}$ en desayuno y cena y $400 \mathrm{mg}$ en comida, junto con Lamotrigina, 100 mg cada 12 horas y Fenobarbital $100 \mathrm{mg}$, cada 12 horas. Cirugías previas: Colecistectomizada. En febrero de 2002 experimenta episodios de labilidad emocional, siendo diagnosticada de depresión exógena, reactiva a problemática familiar, que parecen estar en relación con un incremento del número de crisis comiciales. Ante el irregular control de las mismas, se decide añadir al tratamiento previo, Levetirazetam a dosis de $1.000 \mathrm{mg}$ dos veces al día. Dado el progresivo agravamiento de la clínica psiquiátrica, se decide, finalmente, instaurar tratamiento antidepresivo y ansiolítico con Alprazolam $2 \mathrm{mg}$ cada 8 horas, Venlafaxina $150 \mathrm{mg}$ diarios y Mirtazapina 1 comprimido por las noches, con respuesta satisfactoria.

En septiembre de 2006, la paciente sufre cuadro sincopal, acompañado de hipertonía axial y apendicular, sin relajación esfinteriana y con traumatismo craneal asociado. No refiere factores precipitantes previos (estrés, falta de sueño, etc.) y presenta una adecuada adherencia al tratamiento (niveles de droga en sangre dentro de los límites de la normalidad).

En la exploración física destaca, únicamente, herida inciso-contusa en región frontal derecha. Neurológicamente, en el momento de la asistencia, se muestra obnubilada, sudorosa, pálida, con frialdad cutánea y progresiva recuperación del nivel de consciencia, sin focalidad neurológica concomitante.

En las exploraciones complementarias:

1. Constantes: TA: 142/76, FC: $67 \mathrm{lpm}, \mathrm{T}^{\mathrm{a}}: 36^{\circ} \mathrm{C}$, Sat. $\mathrm{O}_{2}=99 \%$.

2. ECG: Ritmo sinusal a $67 \mathrm{lpm}$, con QT de 0,44" (QTc $\left(\mathrm{QT} /[\mathrm{RR}]^{1 / 2}=0,46 "\right)$, teniendo en cuenta que el QTc máximo para dicha frecuencia en una mujer es de 0,414" (Fig. 1).

3. Radiología craneal: ausencia de fracturas.

4. Radiología torácica: normal.

5. EEG: En región temporal se aprecia actividad focal de ondas agudas y theta de mediana amplitud, con escasa frecuencia de aparición a lo largo del trazado (similar a los previos).

6. Analítica general: Destaca un sodio de $124,8 \mathrm{mEq} / \mathrm{l}$ (Fig. 2), con un cloro de $88 \mathrm{mEq} / \mathrm{l}$ y leucocitosis (16800 células $/ \mathrm{mm}^{3}$ ) con neutrofilia. Las concentraciones plasmáticas de glucosa, urea, creatinina, potasio, bilirrubina total y fraccionada, así como las transaminasas, estaban dentro del rango de la normalidad.

7. Estudio de coagulación: normal.

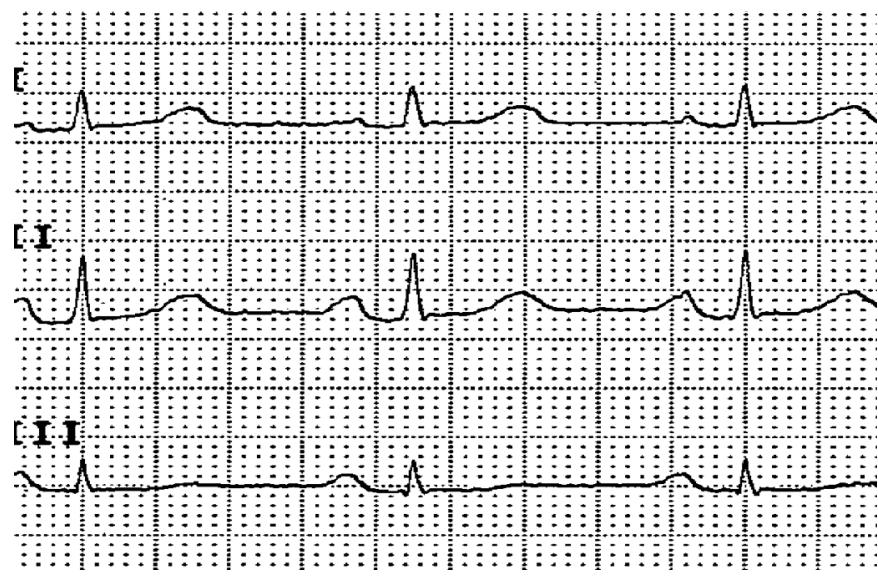

Fig. 1. ECG en el momento del ingreso en nuestro centro, en el que se observan un intervalo QT prolongado $(0,44 \mathrm{seg})$ y un QTC de $0,46 \mathrm{seg}$. 


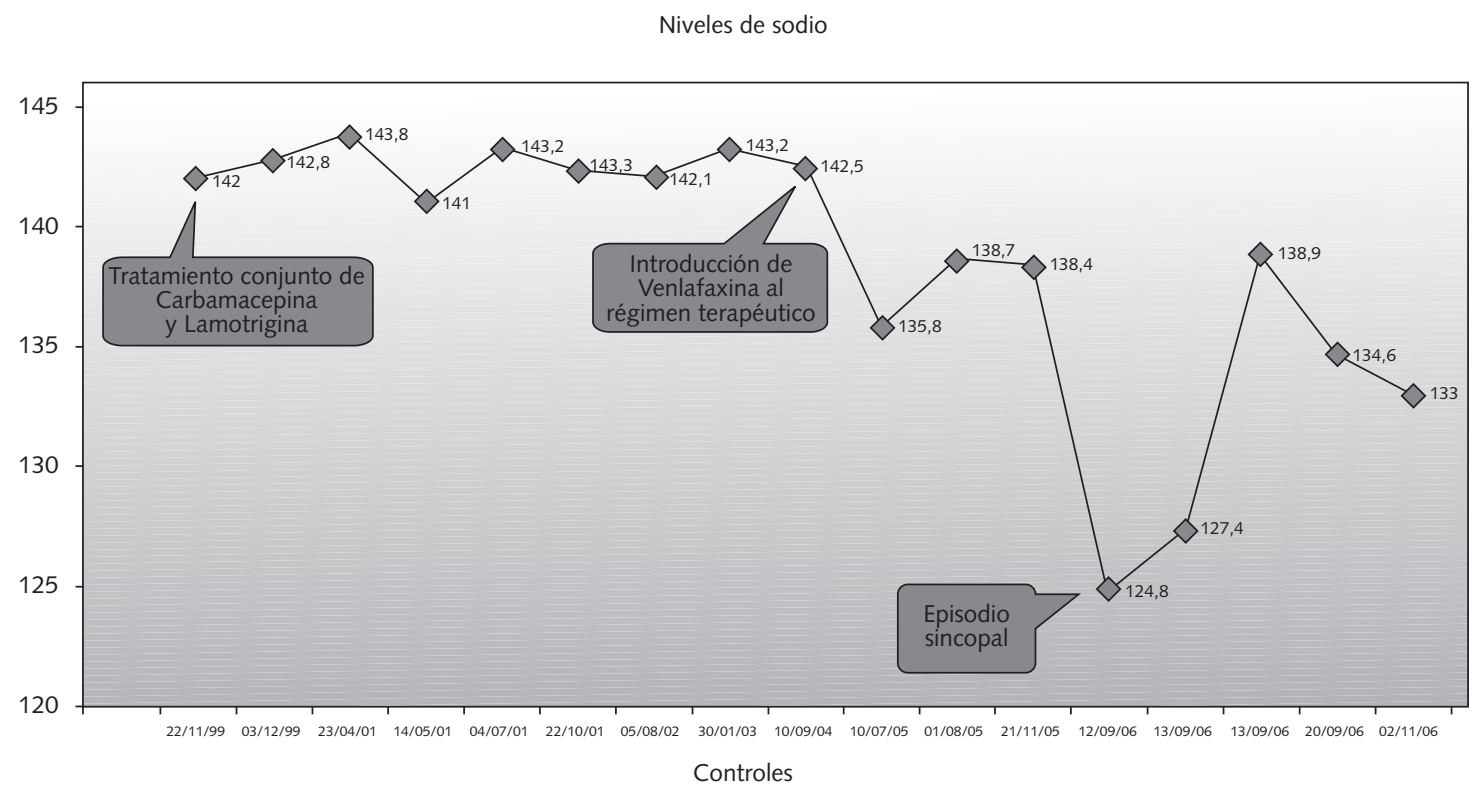

Fig. 2. Evolución de la concentración sérica de sodio en la paciente. Se observa un descenso de los niveles de sodio tras la introducción de la Venlafaxina al tratamiento conjunto con Carbamacepina y Lamotrigina.

8. GV: Niveles de lactato: $2,2 \mathrm{mmol} / \mathrm{l}(0,6-2,2 \mathrm{mmol} / \mathrm{l})$.

9. Niveles de Carbamacepina: 7,4 $\mu \mathrm{g} / \mathrm{ml}$ (4-10 microg/ml).

10. Estudio de autoinmunidad negativo. (11).

11. Estudio de elementos traza: Selenio: $54,6 \mathrm{mg} / \mathrm{l}(46-143 \mu \mathrm{g} / \mathrm{l})$

La paciente ingresa en el Servicio de Urgencias, con el diagnóstico de síncope convulsivo secundario a hiponatremia de probable etiología farmacológica.

Una vez revisada la evolución de las concentraciones séricas de Sodio a lo largo del tratamiento conjunto con Carbamacepina y Lamotrigina, se aprecia una cifra media de 142,5 mEq/1 (141-143,8 $\mathrm{mEq} / \mathrm{l})$. Sin embargo, tras la introducción de la Venlafaxina, el Sodio experimenta un sensible descenso, sostenido en el tiempo, situándose en valores medios de 133,6 mEq/l (124,8-138,9 mEq/l) lo que supone un descenso medio del 6,2\%, siendo la cifra más baja la correspondiente al episodio sincopal $(124,8 \mathrm{mEq} / \mathrm{l})$. En ningún momento existieron niveles de Carbamacepina superiores a los terapéuticos (3,5-12 $\mu \mathrm{g} / \mathrm{ml})$, que justificaran dichas hiponatremias.

\section{DISCUSIÓN}

Aunque no es posible descartar un nuevo episodio comicial en la paciente, debemos destacar la coincidencia entre el episodio sincopal y la concomitancia del tratamiento con Carbamacepina, Lamotrigina y Venlafaxina.

La Carbamazepina es un antiepiléptico empleado en el tratamiento de las crisis epilépticas generalizadas tónico-clónicas y parciales, tanto simples como complejas, incluyendo la posible generalización secundaria $(12,13)$. Su mecanismo de acción es bastante similar al de la Fenitoína, disminuyendo la entrada de sodio y calcio en la neurona hiperexcitada. Se metaboliza a nivel hepático, generándose dos metabolitos activos, a saber, 10,11-epóxido-carbamazepina y 10,11-dihidróxido-carbamazepina (13). Las concentraciones séricas terapéuticas se sitúan entre 4-10 $\mu \mathrm{g} / \mathrm{ml}$. Entre las reacciones adversas, destacan, sobre el sistema nervioso central, vértigos, cefaleas, ataxia, somnolencia, fatiga y diplopía. A nivel gastrointestinal puede producir náuseas y vómitos. En el ámbito dermatológico son frecuentes las reacciones alérgicas. En cuanto al sistema endocrino y metabólico, es de relevante importancia, en nuestro caso, la hiponatremia e hipoosmolalidad plasmáticas, generadas mediante un mecanismo de secreción inadecuada de hormona antidiurética (SIADH), pudiendo llegar a producir, letargia, vómitos, cefaleas, confusión mental y trastornos de la conducción cardíaca $(14,15)$. Estos efectos adversos se han identificado entre el 5 y el $40 \%$ de los pacientes tratados $(14,16)$. En cuanto a la Lamotrigina, se trata de un nuevo fármaco anticomicial que actúa sobre los canales neuronales de sodio y calcio, disminuyendo su conductividad. Respecto a la actividad recaptadora de Serotonina de la Venlafaxina (inhibidor selectivo mixto de la recaptación de Serotonina y Noradrenalina), al igual que otros representantes del grupo, como la Fluoxetina y la Paroxetina, presentan una vía de metabolización hepática común con la Carbamacepina, mediada por el Citocromo P450, lo que puede conducir a la elevación de las concentraciones plasmáticas de dicho anticomicial, acentuando su efecto hiponatremiante, además de presentar efecto antidiurético propio $(16,17)$.

Parece probable, que en nuestra paciente, haya habido una acción sumatoria de los efectos antidiuréticos e hipoosmolares de Carbamacepina, Lamotrigina y Venlafaxina, lo que condujo a un cuadro sincopal por hiponatremia sostenida.

Consideramos importante destacar el papel del Selenio como cofactor en numerosas enzimas con función antioxidante, que según las hipótesis actuales, desarrollarían un papel esencial como quelantes de radicales libres (18) bloqueando la peroxidación lipídica neuronal que se desarrolla en el foco comicialógeno, por tanto, presentaría efecto neuroprotector. Esta hipótesis está basada en los estudios de Remaekers et al, según el cual, una alteración en el metabolismo del Selenio 
podría actuar como agente desencadenante de convulsiones (19). Niveles descendidos mantenidos en el tiempo pueden explicar convulsiones intratables (20). En nuestra paciente, los niveles de Selenio se encuentran en el límite bajo de la normalidad, lo cual vendría a apoyar su cada vez más relevante papel en la etiología de la epilepsia y su tendencia a la refractariedad.

Por tanto, evitar el daño cerebral propio de la encefalopatía epiléptica, los accidentes, la repercusión psicosocial y la muerte súbita, son las principales justificaciones para el tratamiento intensivo en los pacientes con epilepsia farmacorresistente. Pero este hecho, puede acompañarse de hiponatremia por un mecanismo de SIADH. Por ello, propugnamos en la medida de lo posible, evitar la combinación de anticomiciales como Carbamacepina y Lamotrigina, con antidepresivos del grupo de los ISRS, particularmente Venlafaxina, Fluoxetina y Paroxetina, o si esto no fuese posible, un

\section{Bibliografía}

1. Nashef L. Sudden unexpected death in epilepsy: Terminology and definition. Epilepsia 1997; 38 (Supl. 11): S6-S8.

2. Oppenheimer S. Cardiac dysfunction during seizures and the sudden epileptic death syndrome. J R Soc Med 1990; 83: 134-6.

3. Hauser WA, et al. Mortality in patients with epilepsy. Epilepsia 1980; 21: 399-412.

4. Jones MW. Consequences of epilepsy: Why do we treat seizures? Canadian J Neurological Science 1998; 25 (Supl. 4): 24-6.

5. Timmings PL. Sudden unexpected death in epilepsy: is carbamazepine implicated? Seisure 1998; 7: 289-91.

6. Gordon N. The long Q-T syndromes. Brain and Development 1994; 16: 153-5.

7. Tavernor SJ, Brown SW, Tavernor RME, Gifford C. Electrocardiograph QT lengthening associated with epileptiform EEG discharges- A role in sudden unexplained death in epilepsy? Seizure 1996; 1: 79-83.

8. Langan Y, Nashef L, Sudden unexpected death in epilepsy (SUDEP) ACNR January/February 2003; 2: 6-8.

9. Alegre S, Araujo J, Rubio JM. Interacción claritromicina-carbamacepina: síntomas neurológicos e hiponatremia. An Med Intern 1998; 15: 48-9.

10. Arriba de G, machín JM, Balanza J. Síndrome de secreción inadecuada de ADH durante la administración de paroxetina. Med Clin (Barc) 2003; 121: 158-9.

11. Milne DB. Trace elements. In: Burtis CA, Ashwood ER, editors. Tietz textbook of clinical chemistry, 2 ed. Philadelphia; WB Saunders; 1994.

12. Forcadas I, Zarranz JJ, Prats-Viñas JM, Madoz P. Epilepsias. En: control más estricto del paciente a fin de evitar nuevos episodios sincopales.

Por último, de acuerdo con Ficker (21), consideramos conveniente aportar unas sugerencias para la prevención del SUDEP, como son:

1. Intentar conseguir el mejor control farmacológico posible de los episodios comiciales.

2. Evitar factores desencadenantes o precipitantes de las crisis epilépticas.

3. Asegurarse el adecuado cumplimiento de la terapia anticomicial.

4. Evitar el uso concomitante de cualquier tipo de droga, estimulantes o alcohol.

5. La familia del paciente debería familiarizarse con las medidas de resucitación cardiopulmonar.

6. Informar y educar a la familia sobre el Síndrome SUDEP.

Zarranz JJ. Neurología . $3^{\text {a }}$ ed. Elsevier; 2003. p. 437-99.

13. Matthew RP, Naif ZA Jr. Toxicology and therapeutic drug monitoring. In: McPherson RA , Pincus MR. Henry's Clinical Diagnosis and Management by Laboratory Methods. 21th ed. Saunders; 2007. p 311-3.

14. Madhusoodanan S, Bougnovic OJ, Moise D, Brenner R, Markovitz S, Sotelo J. Hyponatremia associated with psycotropic medications. A review of the literature and spontaneous reports. Adverse Drug React Toxicol Rev 2002; 21: 17-29.

15. Stone S, Lange LS. Syncope and sudden unexpected death attributed to carbamacepine in a 20 year old epileptic. J Neurol Neurosurg Psychiatry $1987 ; 49$ : 1460-1.

16. Martindale. The extra pharmacopoeia. $31^{\text {th }}$ ed. London: Royal Pharmaceutical Society 1996; p. 369-72.

17. Committee on Safety of Medicines/Medicines Control Agency. Antidepressant-hyponatraemia induced. Current Problems 1994; 20: 5-6.

18. Klein EA. Selenium: Epidemiology and basic science. J Urol 2004; 171 : S50-S3.

19. Gutiérrez-Álvarex AM, Moreno CB, González Reyes RE. Alteración de la concentración de selenio en la epilepsia. Rev Neurol 2005; 40: 111-6.

20. Remaekers VT, Calomme M, Vanden Berghe D, Makropoulos W. Selenium deficiency triggering intractable seizures. Neuropediatrics 1994; 25: 217-23.

21. Ficker M. Sudden unexplained death and injury in epilepsy. Epilepsia 2000; 41 (Supl. 2): S7-S12. 